PROC. OF JSCE,

No. 218, OCT. 1973

\title{
THE EQUIVALENT RADIUS OF A SOURCE IN NUMERICAL MODELS OF GROUNDWATER FLOW
}

\author{
By Iichiro KONO*
}

\begin{abstract}
It is shown, by the theoretical consideration and by some numerical verifications, that when a well is incorporated in a network of a numerical model for plane groundwater flow by a simple term in the continuity equation for a node, this can be considered to mean that the well is supposed to have a certain radius, the value of which is a constant times of the mesh width. Especially for the network of square mesh, the equivalent radius is about 0.2 times of the mesh width.
\end{abstract}

\section{INTRODUCTION}

In numerical models for the solution of plane problems of groundwater flow, such as the finite difference method or the finite element method, it is often necessary to include one or more wells. The simplest way of dealing with such wells is to consider the discharge of a well as a supply in a nodal point.

As in most numerical models a system of numerical equations is derived on the basis of a conservation equation in each node, a well in a certain node can be taken into account as a term in the equation for that node, of magnitude equal to the discharge of the well. It can be expected that this leads to satisfactory results at great distances from the well, but the results in the vicinity of the well seem to be doubtful. Especially the values of the groundwater head in the nodes at which wells are operating are hard to interpret as the computer program will yield a finite value, and it is well-known that in a point source or sink the head has to be infinite.

It will be shown in this paper that there exists a simple interpretation for a source or sink in a nodal point of a network of a numerical model for groundwater flow, in terms of an equivalent

* Dr. Eng., Associate Professor, Department of Civil Engineering, Kyoto University. non-zero radius of the well.

\section{THEORETICAL CONSIDERATION}

In the case of a sink of discharge $Q_{p}$ in a certain point, the head of groundwater will be singular near that point, and in plane groundwater flow the singularity is of the logarithmic type. Near the sink (or source) the head is as well-known

$$
h=H-\frac{Q_{p}}{2 \pi T} \ln \left(\frac{R}{r}\right)
$$

where $h$ is the head of groundwater, $T$ is the coefficient of transmissibility, $r$ is the distance from the center of a well, $R$ is the radius of the influent circle and $H$ is the head on the influent circle.

In the immediate vicinity of the sink the head is practically constant, namely the water head of a well. In the case of a well of radius $r_{e}$, the singularity is of same type and the head $h_{0}$ in the well is

$$
h_{0}=H-\frac{Q_{p}}{2 \pi T} \ln \left(\frac{R}{r_{e}}\right)
$$

At a (small) distance $\Delta 1$ from the center of the well the head $h_{i}$ is

$$
h_{i}=H-\frac{Q_{p}}{2 \pi T} \ln \left(\frac{R}{\Delta 1}\right)
$$

From Eqs. (2) and (3) it follows that the head difference $\Delta h_{0}$ between the one of the well and of the point at distance $\Delta 1$ is

$$
\Delta h_{0}=h_{i}-h_{0}=\frac{Q_{p}}{2 \pi T} \ln \left(\frac{\Delta 1}{r_{e}}\right)
$$

The formula (4) shows a linear relationship between the head difference with the surrounding points and the discharge of the well. In a network for numerical calculations there also exists a linear relationship between the both, only of a different character.

(1) In a square mesh as shown in Fig. 1, the formula used is 


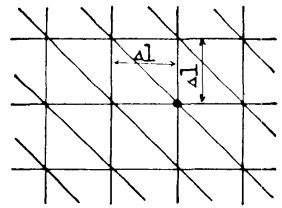

(a) Elements in finite element method

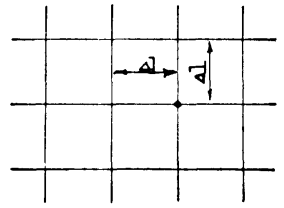

(b) Mesh in finite difference method

Fig. 1 Networks of square mesh type for numerical models.
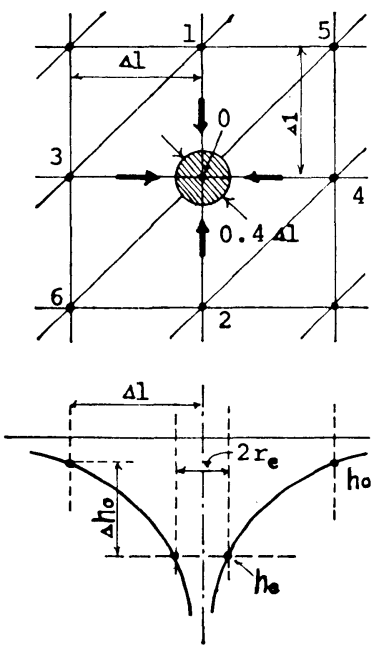

Fig. 2

$$
Q_{p}=4 T \cdot \Delta h_{0}
$$

where the 4 originates from $\left(h_{i}-h_{0}\right),(i=1,2,3,4)$ $h_{1}, h_{2}, h_{3}$ and $h_{4}$ being the values of the head in the 4-neighbouring points, as shown in Fig. 2. [APPENDIX]

The formulas (4) and (5) are equivalent if

$$
\frac{r_{e}}{\Delta 1}=\exp \left(-\frac{\pi}{2}\right) \quad(=0.208)
$$

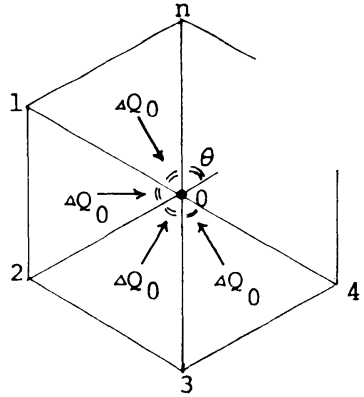

Fig. 3

This means that a point sink in a square mesh for numerical calculations is equivalent to a well of radius $r_{e}=\Delta 1 \cdot \exp \left(-\frac{\pi}{2}\right) \doteqdot 0.2 \Delta 1$ in a continuous flow domain, where the distance $\Delta 1$ is to be interpreted as the mesh width.

The radius $r_{e}$ may be called the equivalent radius of the sink (or source, if $Q_{p}$ is negative).

(2) For other cases of networks similar calculations and interpretations can be made.

In the network as shown in Fig. 3, the point sink 0 surrounded by the $n$-elements of equilateral triangles, the following formula (7) is used in calculating by the finite element method [APPENDIX] instead of Eq. (5).

$$
\left.\begin{array}{l}
Q_{p}=\text { Const } \cdot T \Delta h_{0} \\
\text { Const }=n\left(\frac{1-\cos \theta}{\sin \theta}\right), \quad n=\frac{360^{\circ}}{\theta^{\circ}}
\end{array}\right\}
$$

where $\Delta h_{0}=h_{i}-h_{0}(i=1,2,3, \cdots, n)$.

Const in Eq. (7) depends only on the number of the surrounding triangular elements, namely on the angle of the triangular element at the nodal point 0 .

From Eqs. (4) and (7) the following formula is obtained

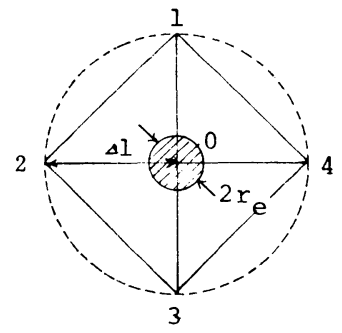

(a) $\mathrm{n}=4$ $r_{e}=0.208 \Delta 1$

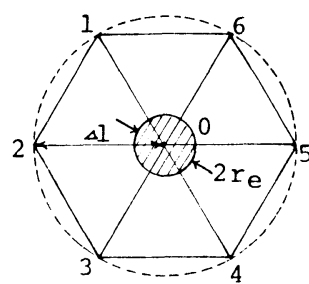

(b) $n=6$ $r_{e}=0.298 \Delta 1$

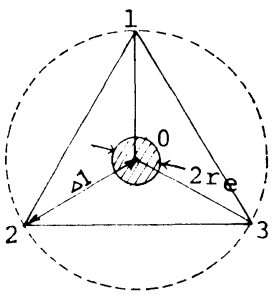

(c) $n=3$ $r_{e}=0.163 \Delta 1$

Fig. 4 Equivalent radius in the networks for finite element method. 


$$
r_{e}=\Delta 1 \cdot \exp \left(-\frac{2 \pi}{\text { Const }}\right)
$$

Hence the following Table 1 and Fig. 4 are gotten from Eqs. (7) and (8).

Table 1

\begin{tabular}{c|c|c|c|c}
\hline$\theta$ & $n$ & Const & $\left(r_{e} / \Delta 1\right)$ & Fig. 4 \\
\hline $60^{\circ}$ & 6 & $2 \sqrt{3}$ & 0.298 & (b) \\
\hline $90^{\circ}$ & 4 & 4 & 0.208 & (a) \\
\hline $120^{\circ}$ & 3 & $3 \sqrt{3}$ & 0.163 & (c) \\
\hline
\end{tabular}

The case of $n=4$ has been discussed in (1). For an arbitrary value of $\theta$ (or $n$ ) the equivalent radius $r_{e}$ is calculated as a constant times of the mesh width.

\section{NUMERICAL VERIFICATION}

In order to verify the formula (6) some numerical calculations were carried out for the case of a well in a homogeneous aquifer.

The size of the network was varied in such a way that the outer boundary of the circular domain (along which the head was supposed to be zero) was located at the distances $2 \Delta 1,3 \Delta 1,4 \Delta 1$, $\cdots, 7 \Delta 1$, where $\Delta 1$ is again the mesh width as shown Fig. 5 and the node of a source was located at the center of the circular domain. The head of the source was supposed to be unit.

The calculations were carried out by means of programs for the finite element method with trianglar element and the finite difference method in square mesh.

The results of the calculations are shown in Fig. 5 and are plotted on logarithmic scale in Fig. 6. It appears that the results are indeed of logarithmic character and that all the straight lines pass through the point: $(s, r)=(0,0.2 \Delta 1)$, where $s$ is the amount of drawdown, which means that in this case the interpretation of the point source as a well of radius: $r_{e} \div 0.2 \Delta 1$ is correct.

\section{DISCUSSION}

\section{(1) Finite Element Method and Finite Difference Method}

For the networks of the square mesh type as shown in Fig. 1(a) and (b), the formulas used in the finite element method and the finite difference method happen to be quite the same. Then the relationship of Eq. (6) is satisfied in both methods.

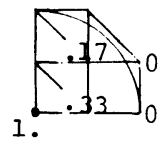

(a) $R=2 \Delta 1$

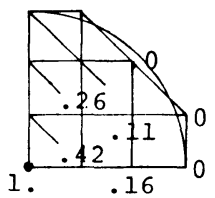

(b) $R=3 \Delta I$

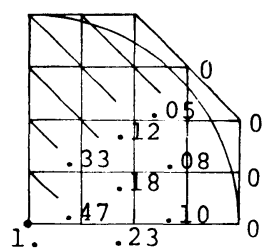

(c) $\mathrm{R}=4 \Delta \mathrm{l}$

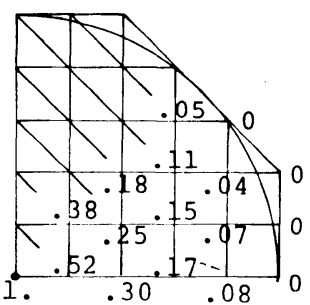

(d) $=5 \Delta 1$

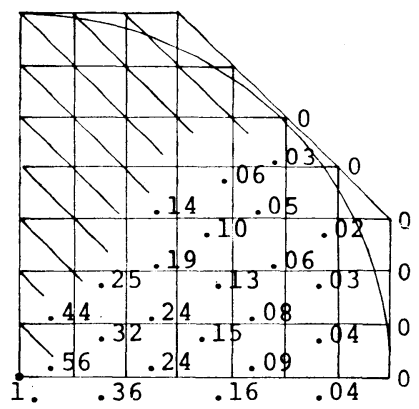

(f) $R=7 \Delta I$

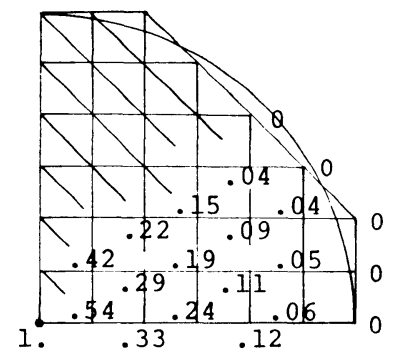

(e) $R=6 \Delta I$

Fig. 5 Drowdowns of head calculated by the finite element method. The drowdowns are supposed to be unit at the node of a well and to be zero at the outer boundary. 


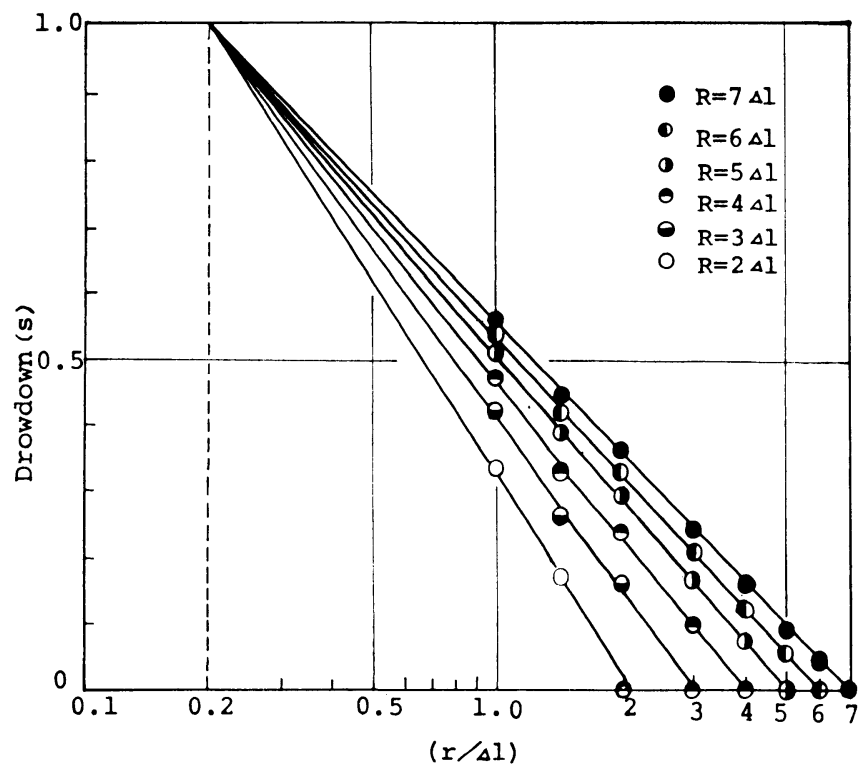

Fig. 6 Relationship between the distance $r$ from the node of a source and the drawdown $s$.

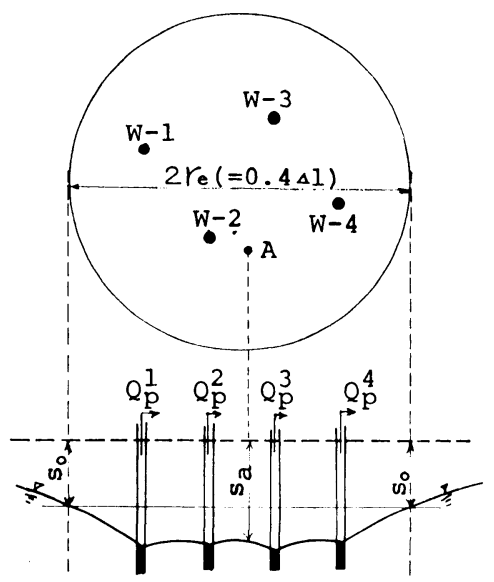

Fig. 7 Multiple wells.
But the general configurations as shown in Fig. 3 are for the finite element method. The finite element method is sometimes more powerful than the finite difference method because in the finite element method can be made arbitrarily shaped triangles as the elements along the boundary, while in the finite difference method is almost limited to the network of square mesh.

\section{(2) Application to Multiple Well System}

In such cases that some wells are located in the vicinity of a node as shown in Fig. 7 and their discharges are $Q_{p}{ }^{1}, Q_{p}{ }^{2}, \cdots, Q_{p}{ }^{n}$ respective$1 \mathrm{y}$, the following calculations are applicable approximately

$$
Q_{p}=\sum_{i=1}^{n} Q_{p}{ }^{i}
$$

where $Q_{p}$ is the amount of water supply at the node as discribed in Eqs. (5), (7) etc.

In the circular region of which the radius is $r_{e}$ in Eqs. (6), (8), the well-known formula of multiple wells is satisfied

$$
\Delta s_{a}=\frac{1}{2 \pi T} \sum_{i=1}^{n} Q_{p}^{i} \ln \left(\frac{r_{e}}{r_{i}}\right)
$$

where $\Delta s_{a}$ is the drawdown at a point $\mathrm{A}$, which is measured with the base of the head at $r=r_{e}$ as shown in Fig. 7, namely the difference between the drawdown $s_{e}$ at $r=r_{e}$ and $s_{a}$ at $A$ and $r_{i}$ is the distance between a point $\mathrm{A}$ and the $i$-well.
Therefore the drawdown $s_{a}$ is

$$
s_{a}=s_{0}+\Delta s_{a}
$$

where $s_{0}$ is gotten by the numerical calculation as already discribed in Sec. 3 .

\section{CONCLUSION}

It has been shown that a point source or a sink in a point of a square mesh for numerical calculations can be interpreted as a well having a radius of a factor 0.2 times (exactly $\exp (-\pi / 2)$ times) of the mesh width. For other types of networks the similar interpretation exists, with a different factor.

\section{[APPENDIX]}

The origin of the cartesian coordinate is put at the node of a point source 0 as shown in Fig. 8. Then the coordinate of the node 1: $\left(x_{1}, y_{1}\right)=$ $(0, \Delta 1)$, the one of the node $2:\left(x_{2}, y_{2}\right)=(\Delta 1 \cos \theta$,

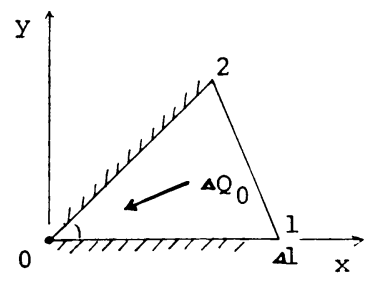

Fig. 8 
$\Delta 1 \sin \theta)$, where $\Delta 1$ is the length of $0-1$ or $0-2$ (mesh width) and $\theta$ is the angle of $1-0-2$ $\left(=360^{\circ} / n, n\right.$ : integer). The following Eq. (12) is used for the element $0-1-2$ in the finite element method

$$
\sum_{j=0}^{2} P_{0 j} h_{j}+\Delta Q_{0}=0
$$

where $P_{i j}$ is the coefficient of the permeability matrix which is called "the stiffness matrix" in structural analysis and $\Delta Q_{0}$ is the amount of the water supply at the node 0 , which is the one limited for the element $0-1-2$.

$$
P_{i j}=\frac{T}{4 \Delta}\left(b_{i} b_{j}+c_{i} c_{j}\right)
$$

where $\Delta$ is the area of the element, $T$ is the coefficient of transmissibility,

$$
\begin{aligned}
& b_{i}=\left(y_{j}-y_{k}\right) \\
& c_{i}=\left(x_{k}-x_{j}\right)
\end{aligned}
$$

$(i, j, k)$ is the cycle permutation of subscripts.

By calculation of $P_{i j}$ with the coordinates of the nodes $(0,1,2)$

$$
\left.\begin{array}{l}
P_{00}=T\left(\frac{1-\cos \theta}{\sin \theta}\right) \\
P_{01}=P_{02}=T\left(\frac{\cos \theta-1}{2 \sin \theta}\right)
\end{array}\right\}
$$

Substitution of Eq. (15) into Eq. (12) gives

$$
\Delta Q_{0}=T\left(\frac{1-\cos \theta}{2 \sin \theta}\right)\left(h_{1}+h_{2}-2 h_{0}\right) \text {. }
$$

The summension of $\Delta Q_{0}$ for all the $n$ elements arround the node 0 is

$$
Q_{p}=\sum_{j=1}^{n} \Delta Q_{0}^{j}
$$

where $Q_{p}$ is the total amount of water supply at the node 0 .

From Eqs. (16), (17) can be obtained the following relationship

$$
Q_{p}=T\left(\frac{1-\cos \theta}{\sin \theta}\right)\left(\sum_{j=1}^{n} h_{j}-n h_{0}\right)
$$

Supposed that $\left(h_{j}-h_{0}\right)$ is constant $\left(\Delta h_{0}\right)$

$$
Q_{p}=n\left(\frac{1-\cos \theta}{\sin \theta}\right) T \cdot \Delta h_{0}
$$

\section{REFERENCES}

1) Matsuo, S. and Kono, I.: Calculation of the Discharge of Groundwater by the Finite Difference Method, Journal of JSSMFE, July 1970, pp. 15-19.

2) Zienkiewicz, O. C. and Cheung, Y. K.: The Finite Element Method in Structural and Continuum Mechanics, McGraw-Hill, 1967, pp. 148-159.

3) Verruijt, A.: Theory of Groundwater Flow, Macmillan, 1970, pp. 62-68.

4) Kono, I.: Analysis of the Seepage through an Earth Dam by the Finite Element Method, Journal of JSSMFE, August, 1972, pp. 13 19. 